\title{
Stress Fracture Tibia among Military Recruits Management by Patellar Tendon Bearing Cast
}

\author{
Khan MSH${ }^{1}$, Karim R2 ${ }^{2}$ Eqbal SM ${ }^{3}$, Masum A4, Kowser ASM 4
}

DOI: https:/ / doi.org/ 10.3329/ jafmc.v15i2.50834

\begin{abstract}
Introduction: Lower limb overuse injuries are common features of military training. Female recruits have significantly higher incidence of fracture than male recruits. In addition to the cost and time of rehabilitation, injury leads to disillusionment among recruits. Conventionally stress fracture tibia is treated with long leg posterior cast for 6-8 weeks but little is known about the length of time required for rehabilitation and to return to pre-injury level.
\end{abstract}

Objectives: To see the outcome of management of stress fracture tibia among military recruits treated with Patellar Tendon Bearing (PTB) cast for 8 weeks and to find out the rehabilitation time to return to pre-injury level.

Materials and Methods: This prospective study was conducted in Combined Military Hospital, Bogura, Bangladesh, from January 2015 to December 2016. Out of 2600 recruits 74 (male 60 females 14) were diagnosed as case of stress fracture tibia during the period. They were treated by PTB cast for 08 weeks and their treatment and rehabilitation to pre-injury level (follow-up) were recorded prospectively.

Results: Total 85 patients were clinically suspected to have stress fracture tibia, out of them $74(87.06 \%)$ patients were finally diagnosed as case of stress fracture. Of these 74 patients 44(59.46\%) were diagnosed by initial $x$-ray and $30(40.54 \%)$ were diagnosed by MRI scan. Prevalence of stress fracture in male was $2.45 \%$ and in female recruits $9.33 \%$, overall prevalence was $2.85 \%$. About 56(75.67\%) patients developed stress fracture in 0-8 weeks of training, mean rehabilitation time to return to pre-injury level was 15.51 weeks, there was no recurrence of stress fracture and all the patients completed 12 months training, none of them was relegated or withdrawn from training on medical ground.

Conclusion: Patellar tendon bearing cast is an effective, technically simple method for treating stress fracture of tibia in military recruits with excellent outcome. This method not only treats the fracture but also prevents depressive disorders.

Key-words: Stress Fracture Tibia, Military Recruits, Patellar tendon bearing cast.

\section{Introduction}

Stress fractures are overuse injuries of bone and may be defined as, partial or complete fracture that results from repetitive application of stress of less strength than that required fracturing a bone in a single load ${ }^{1}$. Stress fracture occurs in the normal bone of a healthy person due to repetitive small bending and compression forces. Bending stress causes deformation of bone and change in remodeling pattern. With repeated stress osteoblastic bone formation cannot keep pace with osteoclastic resorption resulting in a zone of relative weakness leading to cortical breach of bone. Cancellous bones are affected by repeated compressive forces leading to impacted fracture2. Stress fracture is well recognized in military training and athletes and was first reported in 1855 by Breithaupt3 3 . The incidence of stress fractures in military recruits can be as high as $12 \% 4$, as compared with a rate of $21.1 \%$ of elite athletes ${ }^{5}$ and $1 \%$ of the general population ${ }^{6}$. Causes of increased stress normally relate to a sustained increase in training and more commonly present in athletes who are female, have suffered prior osseous injury and in military recruits. Symptoms are usually of insidious onset, occurring on exertion; however, if allowed to progress, pain can be present at rest or on weight bearing. Clinical findings include localized tenderness and indirect pain produced by tibial percussion and provocative Hop test.

Stress fractures are the most common cause of loss of training days, this is among the more severe form of injuries of lower extremity musculoskeletal system and needs prolong period of refraining from physical activities for recovery?. There are three military training centers, one each for infantry, engineer and armoured corps soldiers under treatment coverage of $\mathrm{CMH}$, Bogura. The training comprises of daily parade in hard ground, $3 \mathrm{~km}$ run, jump from different heights, assault course, weeklong different exercises and $16 \mathrm{Km}$ run. Any recruit who sustains injury is kept away from physical training as per recommendation of treating physician.

Conventionally stress fracture in tibia of recruits are treated by long leg back slab for 6-8 weeks, they take complete rest for the said period, which make them depressed due to apprehension of losing job. This makes others reluctant to report sick early in fear of withdrawal from training, ultimately these cases come in advance stage leading to complications and takes prolonged rehabilitation time. They may also develop disuse muscle wasting and osteopenia. I planned for patella tendon bearing walking cast, which doesn't compel the recruit to bed; they can join training programs except physical training for 08 weeks followed by gradual rehabilitation training for 04 weeks. Walking causes fracture site compression and enhances fracture union, prevents muscle wasting and osteopenia as well as less chance of mental depression and apprehension of losing job.

Biomechanics: In tibia the fracture position can vary according to sporting activity, runners usually have mid to distal $1 / 3$, dancers mid $1 / 3$ and jumping athletes (e.g. tennis, basketball and volleyball) proximal $1 / 3$ injuries 8 . The mid and distal diaphysis of the tibia are the most vulnerable region as this is the narrowest cross-sectional area of the bone and has relatively little surrounding musculature to

1. Lt Col Mohammad Shahnewaz Hossain Khan, MBBS, MS, Classified Orthopaedic Surgeon, Combined Military Hospital, Cumilla 2. Brig Gen Rezaul Karim, MBBS, MS, Advisor Specialist and Classified Orthopaedic Surgeon, Combined Military Hospital, Dhaka 3. Maj SM Eqbal, MBBS, MCPS, MS, Classified Orthopaedic Surgeon, Combined Military Hospital, Bogura. 4. Lt Col Abdullah Masum, MBBS, MCPS, DA, FCPS, Classified Specialist in Anesthesiology, Combined Military Hospital, Cumilla 5. Lt Col ASM Kowser, MBBS, MCPS, Graded Specialist in Psychiatry, Aero Medical Institute, BAF, Dhaka. 
dissipate applied forces. Compressive forces causes fracture in medial cortex and tensile force causes fracture in anterolateral cortex with high risk of nonunion, specially if the fracture is longitudinal9.

Pathophysiology: Repetitive, excessive and intensive weight bearing training of military recruits are well recognized for stress fracture. This unaccustomed training leads to acceleration of bone remodeling that exceeds the bone's intrinsic ability to repair itself. Due to insufficient time for the bone to heal micro fractures subsequently creates a bone stress injury or reaction that eventually results in stress fracture ${ }^{10}$. Poor Nutrition, women with female athlete triad comprising eating disorder, functional hypothalamic amenorrhea and osteoporosis, smoking, alcohol intake are at higher risk of stress fracture ${ }^{11}$.

Diagnosis: Stress fracture should be suspected in athletes or military recruits with a recent increase in physical activity or repeated excessive activity with inadequate rest. Insidious onset of pain, particularly on movement is the most common presenting symptom $(81 \%)$ usually there is no specific inciting event or injury ${ }^{10}$. The classical clinical examination findings are focal tenderness, swelling and rise of temperature at the site of fracture ${ }^{12}$. Sometimes diagnosis become challenging. Medial tibial stress syndrome or shin splints which is characterized by diffuse non localized tenderness along the posteromedial, mid to distal part of tibia and lack of oedema. Other differential diagnosis includes tendinopathy, nerve or artery entrapment syndrome, compartment syndrome and malignancies like osteosarcoma or Ewing's sarcoma ${ }^{13}$. Plain radiography is the first imaging modality for clinically suspected case of stress fracture. Initially plain radiograph often appears normal, initial sensitivity of plain radiograph is 10-20\% and after 3 weeks 30 $70 \%$. If fracture is evident in plain radiograph further imaging is not required. Other imaging modalities are bone scintigraphy which is highly sensitive (74-100\%) but nonspecific. MRI is more sensitive and specific than bone scan. MRI should be considered when plain radiograph is negative ${ }^{10}$.

\section{Materials and Methods}

This prospective study was conducted in Combined Military Hospital, Bogura from January 2015 to December 2016. All the recruits who were diagnosed as stress fracture tibia during this period was treated by PTB cast for 08 weeks and their treatment and rehabilitation follow-up were recorded prospectively. During this period 2600 recruits started basic military training of 44 weeks duration. Recruits who suffered from pain in lower limbs reported to regimental medical clinic, a physician assessed them and suspected cases were exempted from physical activity for 1-2 weeks, recruits who still then having pain were subsequently referred for orthopaedic consultation. In orthopaedic department plain radiograph was taken on initial reporting, symptomatic patients with negative radiograph underwent MRI scan to exclude stress fracture. Patients were kept exempted from physical training since first consultation until confirmation of diagnosis. In diagnosed cases PTB cast was applied for 8 weeks, they were in training activities except physical training, walked on cast, after 4 weeks the cast was changed and a check/ follow-up radiograph was taken. After completion of 08 weeks, PTB was removed. They were allowed to undergo gradual, progressive and individualized physical training for
04-08 weeks to achieve pre-injury level. Then clinical examination was done and on the basis of that they were allowed to resume regular training.

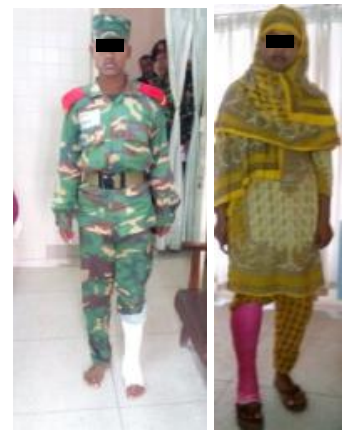

Figure-1: Patients with PTB cast in situ

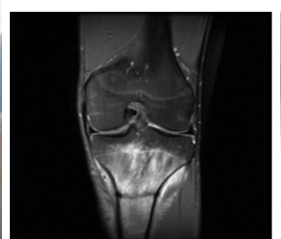

Figure-2: MRI image of stress fracture tibia
Figure-3: X-ray image of stress fracture tibia

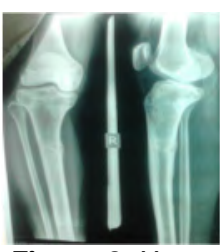

\section{Results}

Among 2600 military recruits, 85 were clinically suspected to have stress fracture tibia, of which $74(87.06 \%)$ were finally diagnosed as case of stress fracture. Overall prevalence of which is $2.85 \%$. Total number of male recruits were 2450 and female 150 . The prevalence in male and female were $2.45 \%$ and $9.33 \%$ respectively. Within this 74 patients 44(59.46\%) were diagnosed by initial x-ray and 30(40.54\%) were diagnosed by MRI scan (Fig-4). Bone scan was not done in any patient. In 74 patients, total 82 stress fractures tibia were detected as 8 patients had bilateral fractures. Two patients had associated distal femoral stress fracture and two had stress fracture in both tibia and fibula. There were 60(81.08\%) male patients and 14 (18.81\%) female patients, age range 17 to20 years, $47(63.5 \%)$ cases in age 17-18 years, $27(36.5 \%)$ cases in age $19-20$ years, no case above 21 years. 60(81\%) cases were from rural area and 14(19\%) from urban area.

Regarding weight at joining in military training $02(2.7 \%)$ were in 45 $50 \mathrm{~kg}, 09(12.16 \%)$ were in $50-55 \mathrm{~kg}, 30(40.54 \%)$ were in $55-60 \mathrm{~kg}$, $14(18.92 \%)$ were in $60-65 \mathrm{~kg}, 4(5.4 \%)$ were in $65-70 \mathrm{~kg}, 4(5.4 \%)$ were in $70-75 \mathrm{~kg}$ group. $20(27.02 \%)$ patients developed stress fracture within 0-1 month, 36(48.65\%) patients in 1-2 months, 06(8.11\%) patients in 2-3 months, 06(8.11\%) patients in 3-4 months, $08(10.81 \%)$ patients in $4-5$ months of joining in military training, no case found after 05 months of joining in military training $100 \%$ of the patients complained of pain on exertion, 54(72.97\%) patients had limping after exertion, 18(24.32\%) patients complained of pain at rest and 12(16.21\%) had local swelling. Clinical examination revealed local tenderness in all cases and they were positive in provocative Hop test. Local swelling was found in 12(16.21\%) cases. 16(21.62\%) patients had bilateral stress fracture tibia. 04(5.4\%) had another bone stress fracture along with tibia ( 02 had stress fracture femur and 02 had stress fracture fibula). 58 (78.38\%) patients had fracture in one leg, among them $36(62.07 \%)$ in right leg and 22(37.93\%) in left leg, $16(21.62 \%)$ had bilateral stress fracture. Stress fractures were more common in middle third of tibia. Distribution of fracture site were proximal third 24(32.43\%), middle third $36(48.64 \%)$, distal third 14(18.91\%), longitudinal fracture 06(8.12\%), transverse fracture 68(91.88\%), postero-medial cortex 66(89.18\%), anteromedial and anterolateral cortex 8(10.82\%).

After treatment $40(54.05 \%)$ patients became symptom free within 0-2 weeks and 32(43.24\%) patients within 2-4 weeks of application 
of PTB. 54(72.97\%) patients returned to pre-injury state within 12-16 weeks, 12 (16.21\%) in 16-20 weeks and 8(10.81\%) in 20-24 weeks (Fig-5). Mean rehabilitation time was 15.51 weeks. There was no recurrence of stress fracture in this series. They were followed up after 01, 02, 03 and 06 months and at the end of training, both clinically and radiologically. All the recruits completed 12 months training, none of them was relegated or withdrawn from training on medical ground.

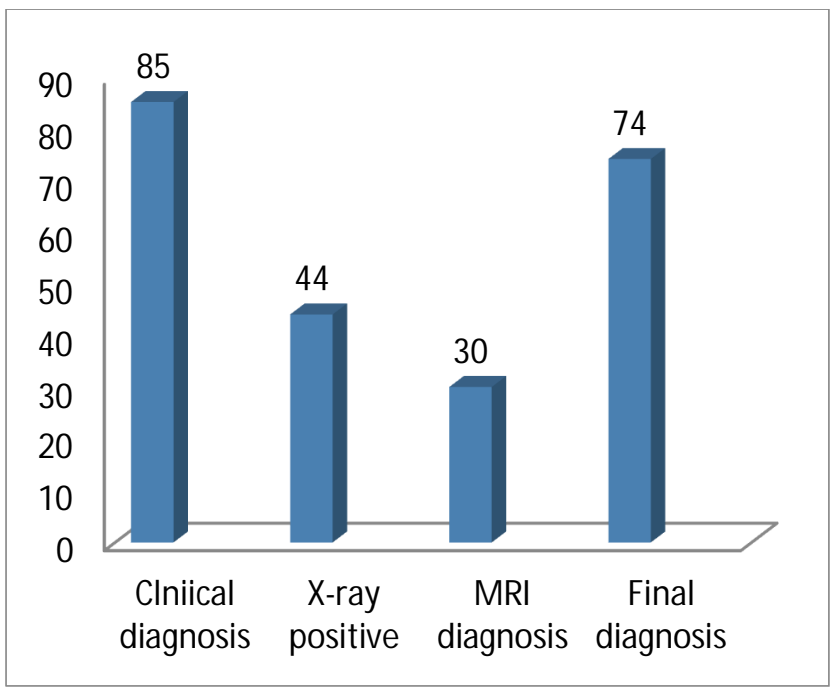

Figure-4: Diagnostic tool of stress fracture tibia patients

\section{Rehabilitation time}

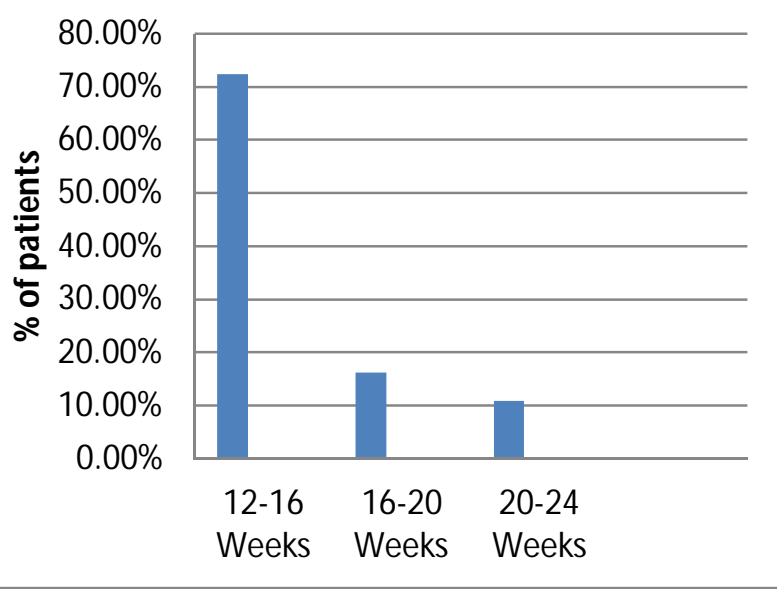

Figure-5: Rehabilitation time of PTB cast treated patients

\section{Discussion}

Stress fractures in military recruits are very common. Military recruits usually lead sedentary lifestyle before joining in basic training unlike athletes, putting the recruits at higher risk for fractures. When new recruits join in military training, running becomes the primary physical training activity and the majority of stress fractures occur in tibia ${ }^{14}$. During the study period 2600 military recruit of 3 training center of Bangladesh army were study population, among them 150(5.7\%)were female. Females are newly recruited in Bangladesh Army only in service corps. Recruits diagnosed as stress fracture tibia was $2.85 \%$, in male recruits prevalence was $2.45 \%$, in female recruits $9.33 \%$ with relative ratio of female male stress fracture 1:3.27. Study conducted in Israel Anti-aircraft Corps from November
1999 to January 2003, revealed that female recruits had significantly more stress ${ }^{15}$ fracture than male recruits with a ratio 2.13. In US army recruits $12.3 \%$ women developed stress fracture compared to $2.4 \%$ in male ${ }^{16}$.

A 04 years Study (2004-2008) conducted by Wood $\mathrm{AM}^{6}$ et al in Royal Marine academy, Lympstone, Devon, showed that prevalence of stress fracture was $5 \%$, among them stress fracture tibia was $24 \%$. In 2002 a study conducted by Ross and All sopp in recruits of basic commando training center of Royal Marine Training Academy showed prevalence of stress fracture was 3.8\% in Revised Common Recruit syllabus (RCRS) and 7\% in original training program. A prospective study conducted in US military undergoing basic training indicated stress fracture incidence of $3.5 \%$ to $8.5 \% 17$. Stress fractures have got multi-factorial etiology, speculation on the precise reason is difficult. Stress fractures and overuse injuries typically occur after muscular fatigue or with sharp change in physical exertion or training, in these situations the capacity of muscles to protect bone from excessive overload is compromised ${ }^{18}$. In Bangladesh, females usually stay in home and do not undergo physical hardship and exercise which make them more prone to develop stress fracture in military training. History of regular exercise is protective against stress fracture. Longer history of exercise further decrease relative risk of fracture ${ }^{18}$. Physiologically progressive training schedule may be adopted to reduce stress fracture.

During the study period 85 patients were clinically suspected to have stress fracture in tibia, $87.03 \%$ were diagnosed as stress fracture tibia, among them $59.46 \%$ were positive in initial X- ray and $40.54 \%$ were diagnosed by MRI scan. In this study, patients who were suspected to have stress fracture tibia in clinical examination, radiograph was taken, positive case underwent treatment protocol and negative cases were selected for MRI for exclusion of stress fracture. We did not wait for the second $x$-ray to be done after 02 weeks, as this may cause worse consequences of delayed diagnosis. High index of clinical suspicion and early MRI for X-ray negative cases may enable prompt diagnosis, reduce rehabilitation time and increase training efficacy. $48.65 \%$ recruits were diagnosed to have stress fracture in first $04-08$ weeks of training, $27.03 \%$ in 0 4 weeks, $16.22 \%$ in $8-16$ weeks and $10.8 \%$ in $16-20$ weeks. Initial 2 months of training is vulnerable for stress fracture, so during these period training activities should be more physiologically progressive specially for female recruits.

Majority of stress fractures occur within first 4-8 weeks, during this early phase of bone remodeling bone resorption temporarily exceeds the ability to withstand increased force ${ }^{6}$. About $27.02 \%$ patients reported for orthopaedic consultation within 1-2 weeks, $48.65 \%$ reported within $2-3$ weeks and $16.22 \%$ reported after $3-4$ weeks of onset of symptoms. Initial $X$ - ray negative patients were relatively early reporters and were confirmed by MRI. These patients had early recovery and returned to pre-injury state within 12 weeks' period. Those who reported late, were in bad shape, $5.4 \%$ of them required 04 weeks initial long leg full plaster in addition to PTB for 8 weeks. So, high index of clinical suspicion, early referral for orthopaedic consultation is very important for early diagnosis and recovery.

Stress fractures were more common in middle third of tibia. Distribution of fracture site were proximal third $32.43 \%$, middle third $48.64 \%$, distal third $18.91 \%$, longitudinal fracture $8.12 \%$, transverse 
fracture $91.88 \%$, postero-medial cortex $89.18 \%$, anteromedial and anterolateral cortex $10.82 \%$. There is a higher incidence of posteromedial cortex fracture in comparison to antero-medial cortex; $54(72.97 \%)$ patients returned to pre-injury state within 12-16 weeks, $12(16.21 \%)$ in 16-20 weeks and 8(10.81\%) in 20-24 weeks, mean rehabilitation time was 15.51 weeks. Study conducted at the Royal Marine Commando Training Center in Lympstone, Devon (20042008) showed rehabilitation time for stress fracture tibia 21.1 weeks ${ }^{6}$. Behrens et al recommended, patients with tibial stress fracture should be exempted from all high impact physical activities until they are asymptomatic when walking ${ }^{19}$. Regardless of the form of treatment, return to physical activity must be gradual and individualized for each patient to prevent subsequent injury20. Patients were followed up for 24-36 weeks. In first 12 weeks; 4 weekly, then 12 weekly and at the end of training. Mean follow up time was 26.3 weeks.

In this study none of the female recruits had any menstrual disturbance, number of menstrual periods were between 11 to 12 per year. Age at menarche and frequency didn't predict stress fracture. However, published data suggests amenorrhea and oligomenorrhea are associated with osteopenia and stress fracture ${ }^{18}$. There was no recurrence of stress fracture in this series. It is recognized that cause of stress fracture is multi-factorial, prevention is difficult but prevalence can be reduced by modification of training program. Fundamental of treatment remains early identification of symptoms, early diagnosis, sufficient long pause in physical training to prevent re-fracture.

\section{Conclusion}

Female recruits are more prone to stress fracture in mixed gender training schedule. There should be different training schedule according to gender in which female recruits would have a lower level of target strain. Patellar tendon bearing cast is an effective, technically simple method for treating stress fracture in tibia in military recruits with excellent outcome. This method not only treats the fracture but also prevents depressive disorders and apprehension to lose the job as they can join all the training programs except physical training in the 08 weeks treatment period. Stress fracture is significant burden in recruit training; they account for long rehabilitation time as well as costs of treatment and training. This warrants further research into early detection, prevention, recovery and rehabilitation time.

\section{References}

1. Ivkovic A, Bojanic I, Pecina M. Stress fractures of the femoral shaft in athlets: A new treatment algorithm. Br J Sports Med 2006; 40: $518-20$.

2. Nayagam S. Principles of Fracture. In Solomon L, Warwick D, Nayagam S. Apley's System of Orthopaedics and Fractures. 9th ed.: Hodder Arnold 2010:724.

3. Lacroix $\mathrm{H}$, Keeman JN. An unusual stress fracture of the fibula in a long distance runner. Archives of Orthopaedics and Trauma 2010; 3(5): 289-290.
4. Protzman RR, Griffis GC. Stress fractures in men and women undergoing military training. The Journal of Bone and Joint Surgery 1977; 59(6):825.

5. Bennell KL, Malcolm SA, Thomas SA. Risk factors for stress fractures in track and field athlets: a twelve months prospective study. American Journal of Sports Medicine 1996; 24(6):810-8.

6. Wood AM, Hales R, Keenan A et al. Incidence and Time to Return to Training for Stress Fractures during Military Basic Training. Journal of Sports Medicine 2014:1-5.

7. Friedl K, Nuovo J, Patience T, Dettori J. Factors associated with stress fracture in young army women: Indications for further research. Military Medicine 1992; 157(7):334.

8. Batt M. Acute and overuse injuries of leg and calf. In Vanhoenacker Fm, Mass M, Gielen JL, editors. Imaging of Orthopaedic Sports Injuries. Springer 2007:355.

9. Edwards PH, Wright ML, Hartman JF. A Practical Approach for the Differential Diagnosis of Chronic Leg Pain in the Athelet. Am. J. Sports Med 2005; 33:1241.

10. Ai-Khawashki $H$. Stress fractures in athletes: $A$ literature review. Saudi Journal of Sports Medicine 2015; 15(2):123-6.

11. Nattir A, Loucks AB, Manore MM et al. American College of Sports Medicine position stand. The female athlete triad. Med Sci Sports Exerc 2007; 39(10):1867-82.

12. Clement DB, Ammann W, Taunton JE et al. Exercise induced stress injuries to the femur. Int J Sports Med 1993; 14(6): 347-52.

13. Fayad LM, Kamel IR, Kawamoto S, Bluemke DA. Distinguishing stress fracture from pathologic fractures: A multimodality approach. Skeletal Radiol 2005; 34(5):245-59.

14. Bowden G, McNally M, Thomas S. Oxford Handbook of Orthopaedics and Trauma: Oxford: Oxford university Press; 2013.

15. Bar-Dayan Y, Gram A, Goldstein L et al. Comparison of stress Fractures of Male and Female Recruits during Basic Training in the Israeli Anti-Aircraft forces. Military Medicine 2005; 170(8):710-2.

16. Wentz L, Liu PY, Haymes E et al. Females have greater incidence of stress fractures than males in both military and athletic populations:A systemic review. Military Medicine 2011; 176(4):420-30.

17. Warden SJ, Burr DB, Brukner PD. Stress Fractures: Pathophysiology, Epidemiology, and Risk Factors. Current Osteoporosis Reports 2006; 4:103-9.

18. Lappe JM, stegman MR, Recker RR. The Impact of Lifestyle Factors on Stress Fractures in Female Army Recruits. Osteoporosis Int 2001; 12:35-42.

19. Behrens SB, Deren ME, Matson A. Stress fracture of the pelvis and legs in athlets: a review. Sports Health 2013 March; 5(2):165-74.

20. Edwards PH, Wright ML, Hartman JF. A Practical Approach for the Differential Diagnosis of Chronic Leg Pain in the Athlets. American Journal of Sports Medicine 2005; 33(8):1241-9. 